ESAIM: PROCEEDINGS, February 2007, Vol.16, 114-132

Eric Cancès \& Jean-Frédéric Gerbeau, Editors

DOI: $10.1051 /$ proc:2007015

\title{
NEAR-WALL SCALING FOR INCOMPRESSIBLE AND COMPRESSIBLE FLOWS
}

\author{
Peller, N. , Manhart, M. ${ }^{1}$, Petrovan Boiarciuc, M. And Brun, C. ${ }^{2}$
}

\begin{abstract}
The paper presents near-wall scaling in incompressible and compressible flows. It concentrates on flows with streamwise pressure gradients which render traditional scaling inapplicable. For incompressible flows a scaling for the velocity profiles is introduced based on both the friction velocity and the pressure gradient. For compressible flows a scaling is developed for the velocity and the temperature. This scaling is based on wall friction, pressure gradient and wall heat flux. We define two new parameters $\alpha$ and $\beta$ where $\alpha$ defines a measure for the ratio between wall friction and pressure gradient and $\beta$ defines the ratio between wall heat flux and pressure gradient. For zero pressure gradient flows the traditional scaling is recovered. A priori tests are performed by means of Direct Numerical Simulations (DNS). The compressible scaling is analyzed on channel flow with non-adiabatic walls and adverse pressure gradient. The incompressible scaling is analyzed on channel flow with periodic hill constrictions. Finally a wall model based on the new scaling is tested a posteriori by means of Large Eddy Simulation (LES).
\end{abstract}

\section{INTRODUCTION}

Large Eddy Simulation (LES) of high Reynolds number wall bounded flows suffers from the fact that the nearwall production mechanisms escape the usual subgrid scale (SGS) modeling [1]. To capture these mechanisms, a grid sufficiently fine to resolve the involved eddies is necessary. Recommendations for near-wall resolution requirements in 3 dimensions have been given by several authors [2-4]. Normally, in the case of attached flows the term wall resolved LES is used when the first grid point lies at $y^{+} \approx 1$. Unfortunately, the ratio between inner scaling representing the near-wall dynamics and outer scaling representing the large scale dynamics increases with Reynolds number. Chapman [5] estimates that for the representation of the dynamics of only one percent of the boundary layer thickness degrees of freedom in the order of $O\left(R e^{1.8}\right)$ are required. For comparison he states that for the outer flow only $O\left(R e^{0.4}\right)$ degrees of freedom are necessary. When modeling the near-wall physics the no-slip boundary condition is not sufficient any more. Models must be applied to the boundary velocity or gradients such as e.g. wall shear stress. This reduces the degrees of freedom greatly and the first grid point can be placed between $20 \leq y^{+} \leq 200$ [1] thus reducing the numerical effort substantially. Nevertheless modeling the complex dynamics of the boundary layer requires detailed investigation on the underlying physics. The basis for this information is provided by Direct Numerical Simulations (DNS).

The design of explicit wall models, i.e. when no special transport equations based on simplified boundary layer equations are solved, is dependent on some universal behaviour of velocity profiles close to the wall. If such an universal behaviour was known, then the layer described by this universal behaviour can be bridged in

\footnotetext{
1 Fachgebiet Hydromechanik, Technische Universität München, Arcisstrasse 21, 80333 München, Germany, email: n.peller@bv.tum.de, m.manhart@bv.tum.de

2 Laboratoire de Mécanique et d'Energétique, Polytech'Orléans, 8 rue Léonard de Vinci, 45072 Orléans France, email: margareta.petrovan@univ-orleans.fr, christophe.brun@univ-orleans.fr
} 
the simulation leading to considerable savings of CPU time and memory requirements. The search for universal near wall behaviour is therefore one of the key elements in designing efficient solvers for high Reynolds number flows in complex geometries.

\section{Analytical LaW of the Wall and NeAR-WALl SCALing}

A universal near-wall behaviour can only be found when velocity profiles can be matched by applying a scaling law. Such a near-wall scaling is desired for compressible and incompressible flows. Whereas in the incompressible case only the velocity profiles need to be scaled, in the compressible case a scaling law must be found for the temperature and the velocity taking into account the changes in density and viscosity. Two different types of near-wall flows can be distinguished for both cases, flows with and without streamwise pressure gradient. For incompressible flows when no pressure gradient is present such as in e.g. zero pressure gradient boundary layers, the region in the vicinity of the wall is mainly governed by the wall shear stress. Dimensional considerations lead to the classical law of the wall based on inner coordinates. In compressible flows, however, the inner coordinates are traditionally based on (i) the wall shear stress and on (ii) the wall heat flux. When moving to more complex flow situations with streamwise pressure gradient these well known laws of the wall are no more a valid description of the flow profile in the vicinity of the wall [6-9]. If e.g. a turbulent boundary layer along a flat plate is approaching a line of separation, deviations from the classical law of the wall can be observed well before the mean separation line [10]. Pressure gradient effects are included into the present formulation of the scaling law, for both compressible and incompressible flows.

\subsection{Incompressible scaling including $\partial p / \partial x$}

The task of near-wall scaling has been addressed by several studies taking the streamwise pressure gradient into account. As already stated by Stratford [11-13] a scaling of the velocity profile close to the wall has to be described by both, the wall shear stress and the pressure gradient. Skote and Henningson [14] proposed a wall scaling based on either wall stress or pressure gradient, depending on which one is the larger one. In the contex of wall models for LES, Manhart [15] found out that a combination of both quantities is necessary to predict the instantaneous velocity behaviour close to the wall.

We consider the boundary layer within the viscous flow region including pressure gradient effects. The Navier Stokes equations for the streamwise velocity component reduce then to

$$
-\frac{\partial p}{\partial x}+\frac{\partial}{\partial y}\left[\mu \frac{\partial U}{\partial y}\right]=0
$$

Integration from the wall yields the following parabolic profile [11-13]:

$$
U(y)=\frac{\tau_{w}}{\mu} y+\frac{1}{2 \mu} \frac{\partial p}{\partial x} y^{2}
$$

where $\tau_{w}$ is the wall friction and $\frac{\partial p}{\partial x}$ is the longitudinal pressure gradient (assumed constant in the wall normal direction). Following Simpson [16], we define a pressure gradient based velocity $u_{p}$ in addition to the standard friction velocity $u_{\tau}$. These two reference velocities write :

$$
\begin{aligned}
& u_{\tau}=\left|\frac{\tau_{w}}{\rho}\right|^{1 / 2}=\left|\frac{\mu}{\rho} \frac{\partial U}{\partial y}\right|_{w}^{1 / 2} \\
& u_{p}=\left|\frac{\mu}{\rho^{2}} \frac{\partial p}{\partial x}\right|^{1 / 3}
\end{aligned}
$$


The ratio $\alpha \in[0,1]$ defined by

$$
\alpha=\frac{u_{\tau}^{2}}{u_{\tau}^{2}+u_{p}^{2}}
$$

describes which of the two effects is preponderant. We finally define a combined velocity scale

$$
u_{\tau p}=\sqrt{u_{\tau}^{2}+u_{p}^{2}}
$$

which is unlikely to go to zero and thus prevents singular behaviour in regions where either $\tau_{w}$ or $\frac{\partial p}{\partial x}$ is small. We then derive a universal scaling law (referred to as scaling 2) of the form

$$
U^{*}\left(y^{*}\right)=A \cdot y^{*}+B \cdot y^{* 2}
$$

with:

and

$$
A=\operatorname{sign}\left(\tau_{w}\right) \alpha \quad, \quad B=\operatorname{sign}\left(\frac{\partial p}{\partial x}\right) \frac{1}{2}(1-\alpha)^{3 / 2}
$$

$$
U^{*}=\frac{U}{u_{\tau p}} \quad, \quad y^{*}=\frac{y u_{\tau p}}{\nu}
$$

This relation constitutes a generalisation of the scaling proposed by Simpson. For flows with zero pressure gradient $(\alpha=1.0)$, we obtain the classical law of the wall (referred to as scaling 1 )

$$
U^{+}=y^{+}
$$

with

$$
U^{*}=U^{+} \quad \text { and } \quad y^{*}=y^{+} .
$$

For flows with zero wall friction $(\alpha=0.0)$, we obtain a parabolic law accounting for the pressure gradient effect only :

$$
U^{*}\left(y^{*}\right)=\frac{1}{2} y^{* 2}
$$

For realistic flow configurations, both contributions are blended in equation (2.7). For $\alpha_{b} \approx 0.3$ the factors $A$ and $B$ in equation (2.7) are identical. A parametric study for $\alpha \in[0,1]$ will be undertaken to check the universality of the proposed law of the wall and determine its domain of validity in terms of $y^{*}$.

\subsection{Analytical law for compressible flows}

For compressible boundary layers, density and viscosity changes at the wall have to be taken into account. This is performed e.g. in the so called van Driest transformation [17] for the logarithmic law of the velocity. We propose to extend this concept to the viscous zone of boundary layers with non-zero longitudinal pressure gradient. The starting point are the Navier-Stokes equations written for a $2 \mathrm{D}$ compressible stationary boundary layer:

$$
\begin{array}{r}
-\frac{\partial p}{\partial x}+\frac{\partial}{\partial y}\left[\mu(T) \frac{\partial U}{\partial y}\right]=0 \\
-\frac{\gamma}{\gamma-1} U \frac{\partial p}{\partial x}+\frac{\partial}{\partial y}\left[\lambda(T) \frac{\partial T_{i}}{\partial y}\right]=0
\end{array}
$$

where the total temperature $T_{i}$ is defined as $T_{i}=T+\frac{P r}{2 c_{p}} U^{2}$. 
2.2.1. Wall scaling for compressible boundary layers with zero pressure gradient

First consider an attached boundary layer with zero pressure gradient $\frac{\partial p}{\partial x}=0$. For non-adiabatic boundary layers, there exist strong changes in viscosity and conductivity at the wall. These changes in flow properties in the viscous sublayer are used as weighting factors to define an integral velocity and an integral total temperature [17-20], respectively:

$$
\begin{aligned}
I_{1}^{+} & =\int_{0}^{U^{+}} \frac{\mu(T)}{\mu_{w}} d U^{+}=y^{+} \\
I_{2}^{+}-T_{w}^{+} & =\int_{T_{w}^{+}}^{T_{i}^{+}} \frac{\mu(T)}{\mu_{w}} d T_{i}^{+}=\operatorname{Pr} y^{+}
\end{aligned}
$$

with $U^{+}=U / u_{\tau}, T_{i}^{+}=T_{i} / T_{\tau}$, where the ${ }^{+}$superscript refers to the usual wall unit involving a friction velocity and a friction temperature (based on the wall heat flux $q_{w}$ ) for compressible flows, respectively:

$$
\begin{aligned}
& u_{\tau}=\left|\frac{\tau_{w}}{\rho_{w}}\right|^{1 / 2}=\left|\frac{\mu_{w}}{\rho_{w}} \frac{\partial U}{\partial y}\right|_{w}^{1 / 2} \\
& T_{\tau}=\left|\frac{q_{w}}{\rho_{w} C_{p} u_{\tau}}\right|=\left|\frac{\lambda_{w}}{\rho_{w} C_{p} u_{\tau}} \frac{\partial T}{\partial y_{w}}\right|
\end{aligned}
$$

A similar transformation (referred to as compressible scaling 1) has been proposed [21-23] to define rather an integral lenghscale $y^{c+}$ :

$$
\begin{aligned}
U^{+} & =\int_{0}^{y^{+}} \frac{\mu_{w}}{\mu(T)} d y^{+}=y^{c+} \\
T_{i}^{+}-T_{w}^{+} & =\operatorname{Pr} \int_{0}^{y^{+}} \frac{\mu_{w}}{\mu(T)} d y^{+}=\operatorname{Pr} y^{c+}
\end{aligned}
$$

This integral lengthscale is very practical since it may be used to build a realistic friction Reynolds number for compressible boundary layers $R e_{\tau}^{c}=\int_{0}^{\delta^{+}} \frac{\mu_{w}}{\mu(T)} d y^{+}=\delta^{c+}$ similar to the one proposed by Bradshaw [17] $R e_{\nu}=\sqrt{\frac{\rho_{w}}{\rho}} \frac{u_{\tau} \delta}{\nu_{e}}$ or the semi local-scaling from Coleman et al. [24] $R e_{s l}=\frac{\rho_{e} u_{\tau}^{e} \delta}{\mu_{e}}$.

\subsubsection{Wall scaling for compressible boundary layers including $\partial p / \partial x$}

For separating compressible boundary layers, the longitudinal pressure gradient $\partial p / \partial x$ can not be neglected with respect to wall friction $\tau_{w}=\mu_{w} \frac{\partial U}{\partial y}{ }_{w}$ and wall heat flux $q_{w}=-\lambda_{w} \frac{\partial T}{\partial y}{ }_{w}$ (for non adiabatic wall conditions). Velocity and total temperature are dependent on this set of parameters:

$$
\begin{aligned}
& I_{1}=I_{1}\left(\tau_{w}, \frac{\partial p}{\partial x}, \mu_{w}, y\right) \\
& I_{2}=I_{2}\left(\tau_{w}, q_{w}, \frac{\partial p}{\partial x}, \mu_{w}, \lambda_{w}, y\right)
\end{aligned}
$$


We extend the non-dimensional procedure described in chapter 2.1. and define a pressure gradient based temperature $T_{p}$ and a combined friction/pressure gradient temperature $T_{\tau p}$, respectively:

$$
\begin{aligned}
T_{p} & =\frac{u_{p}^{2}}{2 C_{p}} \\
T_{\tau p} & =T_{p}+T_{\tau}
\end{aligned}
$$

The ratio $\beta \in[0,1]$ defined by

$$
\beta=\frac{T_{\tau}}{T_{\tau}+T_{p}}
$$

describes which of the two effects, wall heat flux and pressure gradient, is preponderant. After integration from the wall, equations (2.13) and (2.14) write:

$$
\begin{aligned}
\frac{\mu(T)}{\mu_{w}} \frac{\partial U^{*}}{\partial y^{*}} & =\operatorname{sign}\left(\frac{\partial p}{\partial x}\right)(1-\alpha)^{3 / 2} y^{*}+\operatorname{sign}\left(\tau_{w}\right) \alpha \\
\frac{\mu(T)}{\mu_{w}} \frac{\partial T_{i}^{*}}{\partial y^{*}} & =\frac{2 \gamma}{\gamma-1} \operatorname{sign}\left(\frac{\partial p}{\partial x}\right)(1-\beta) \sqrt{1-\alpha} \operatorname{Pr} \int_{0}^{y^{*}} U^{*} d y^{*} \\
& +\operatorname{sign}\left(-q_{w}\right) \beta \sqrt{\alpha} \operatorname{Pr}
\end{aligned}
$$

with $U^{*}=U / u_{\tau p}, T_{i}^{*}=T_{i} / T_{\tau p}$ and $y^{*}=\rho_{w} y u_{\tau p} / \mu_{w}$. Integration of equation (2.26) from the wall (without taking into account the sign of $\partial P / \partial x$ and $\tau_{w}$ ) leads to a parabolic velocity profile (referred to as compressible scaling 2):

$$
U^{*}\left(y^{*}\right)=\alpha y^{c *}+\frac{(1-\alpha)^{3 / 2}}{2} y_{2}^{c * 2}
$$

where

$$
\begin{array}{r}
y^{c *}=\int_{0}^{y^{*}} \frac{\mu_{w}}{\mu(T)} d y^{*}=\frac{\mu_{w}}{\mu_{1}} y^{*} \\
y_{2}^{c *^{2}}=\int_{0}^{y^{* 2}} \frac{\mu_{w}}{\mu(T)} d y^{*^{2}}=\frac{\mu_{w}}{\mu_{2}} y^{*^{2}}
\end{array}
$$

are based on the integral lengthscale definition of equation (2.19). Equations (2.29) and (2.30) are used to compute the term

$$
\int_{0}^{y^{*}} U^{*} d y^{*}=\alpha \frac{\mu_{w}}{\mu_{1}} \frac{y^{*^{2}}}{2}+\frac{(1-\alpha)^{3 / 2}}{2} \frac{\mu_{w}}{\mu_{2}} \frac{y^{*^{3}}}{3}
$$

Finally, integration of equation (2.27) from the wall (without taking into account the sign of $\partial P / \partial x$ and $q_{w}$ ) leads to a polynomial total temperature profile (referred to as compressible scaling 2):

$$
\begin{aligned}
T_{i}^{*}\left(y^{*}\right) & =T_{w}^{*}+\beta \sqrt{\alpha} \operatorname{Pr} y^{c *} \\
& +\frac{\gamma}{\gamma-1}(1-\beta) \operatorname{Pr}\left[\frac{\alpha \sqrt{1-\alpha}}{3} \frac{\mu_{w}}{\mu_{1}} y_{3}^{c *^{3}}+\frac{(1-\alpha)^{2}}{12} \frac{\mu_{w}}{\mu_{2}} y_{4}^{c *^{4}}\right]
\end{aligned}
$$


with

$$
\begin{aligned}
& y_{3}^{c *^{3}}=\int_{0}^{y^{* 3}} \frac{\mu_{w}}{\mu(T)} d y^{*^{3}}=\frac{\mu_{w}}{\mu_{3}} y^{*^{3}} \\
& y_{4}^{c *^{4}}=\int_{0}^{y^{* 4}} \frac{\mu_{w}}{\mu(T)} d y^{*^{4}}=\frac{\mu_{w}}{\mu_{4}} y^{*^{4}}
\end{aligned}
$$

Relations (2.28) and (2.32) constitute a generalisation to compressible flows of equation (2.7). For flows with zero pressure gradient $(\alpha=\beta=1.0)$, we obtain the classical law of the wall for compressible boundary layer equations (2.19) and (2.20) with $U^{*}=U^{+}, T_{i}^{*}=T_{i}^{+}$and $y^{c *}=y^{c+}$. For flows with zero wall friction $(\alpha=0.0)$ and zero wall heat flux $(\beta=0.0)$, we obtain a quadratic law accounting for the pressure gradient effect only (equivalent to equation (2.12) for incompressible flows):

$$
\begin{aligned}
U^{*}(y) & =\frac{1}{2} y_{2}^{c * 2} \\
T_{i}^{*}(y) & =T_{w}^{*}+\frac{1}{12} \frac{\gamma}{\gamma-1} \frac{\mu_{w}}{\mu_{2}} \operatorname{Pr} y_{4}^{c * 4}
\end{aligned}
$$

\section{A priori investigations based on Direct Numerical Simulation (DNS)}

In the following the derived scaling laws will be investigated in flows with and without pressure gradients. For the compressible case a $2 \mathrm{D}$ channel flow is presented. Differences between a periodical channel flow without pressure gradient and a spatially developing channel flow with pressure gradient are pointed out. For the incompressible case channel flow with constrictions is considered. The constrictions are smoothly contoured hills which induce pressure gradients and separation. The pressure gradient effects in this case will be studied in the following.

\subsection{Incompressible channel flow with constrictions}

The numerical setup has been introduced by Mellen et al. [25]. Based on hill height $h$, the channel extends $9.0 h$ in streamwise, $4.5 h$ in spanwise and $3.035 h$ in wall-normal direction. The hills are arranged periodically at the distance 9.0h. In streamwise and spanwise direction periodic boundary conditions are used. On the hill surface a no-slip condition is applied as well as on the top of the channel wall. The configuration can be seen in Figure 1 which shows a snapshot of the streamwise velocity and gives an idea of the complexity of the flow [26,27]. The Reynolds number based on the bulk velocity and the hill height is $R e=5600$. Figure 2 shows the time averaged flow field. The wall shear stress of the time averaged flow field is plotted in Figure 3 . The main separation and reattachment points are found at $x / h \approx 0.17$ and $x / h \approx 5$. A second tiny recirculation zone is present at $x / h \approx 7.2$. A considerable negative wall shear stress exists at $x / h \approx 3.0$ within the recirculation bubble which shows that a strong backflow develops in the recirculation zone. Due to the curvature also a strong streamwise pressure gradient evolves at the lower channel wall (Figure 4).

In order to resolve the complexity of the flow field great effort has been put in the grid design for the DNS [26, 28]. Starting from coarse grid simulations the grid spacings have been determined based on the Kolmogorov scale and the wall shear stress. Table 3.1 gives the grid spacings and resulting number of cells. The computation was performed on the super computer Hitachi SR8000 at the Leibniz Computing centre of Munich with our fully MPI parallelized code [29].

\begin{tabular}{|l|l|l|l|l|l|l|l|}
\hline Re-number & $\Delta x^{+}$ & $\Delta y^{+}$ & $\Delta z^{+}$ & $n x$ & $n y$ & $n z$ & $n x * n y * n z$ \\
\hline 5600 & 8 & 6.3 & 1.2 & 640 & 484 & 753 & $233 \cdot 10^{6}$ \\
\hline
\end{tabular}

TABLE 1. Performed DNS simulation; resolution given for point of maximum wall shear stress 


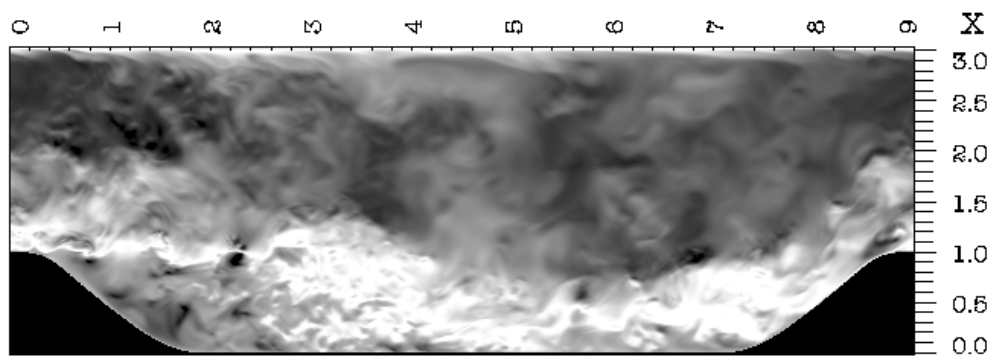

FIGURE 1. Instantaneous streamwise velocity (isolines).

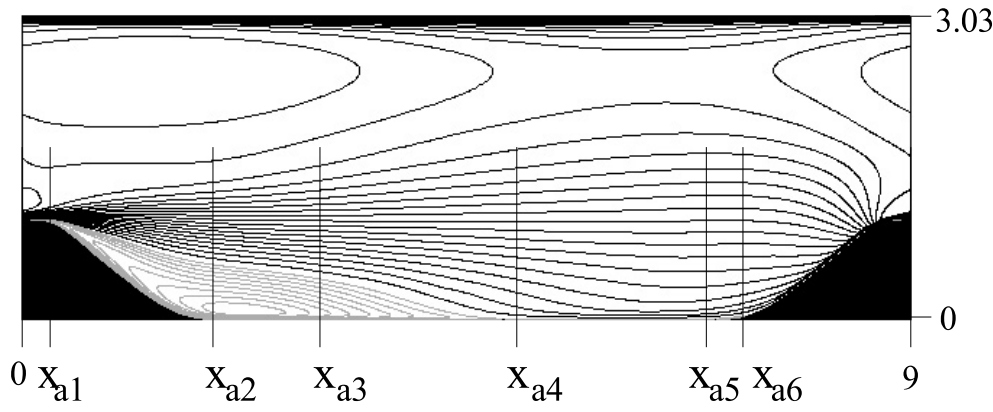

FIgURE 2. Averaged streamwise velocity; black lines: forward flow; grey lines: backward flow

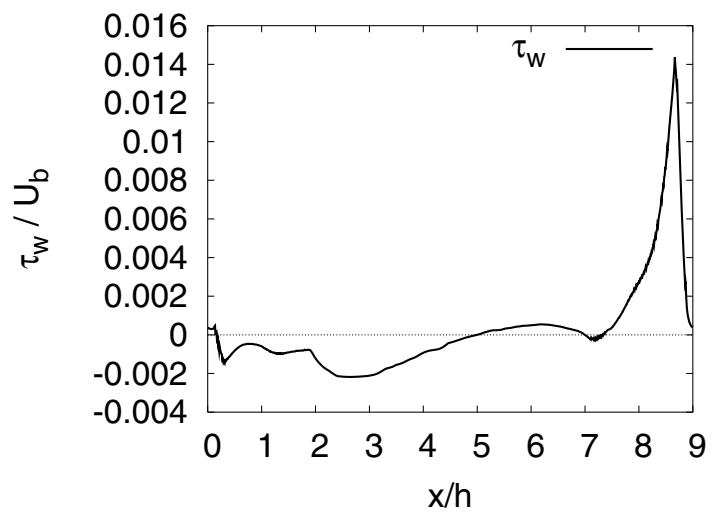

Figure 3. $R e=5600$; Wall shear $\tau_{w}$ stress plotted along the bottom wall.

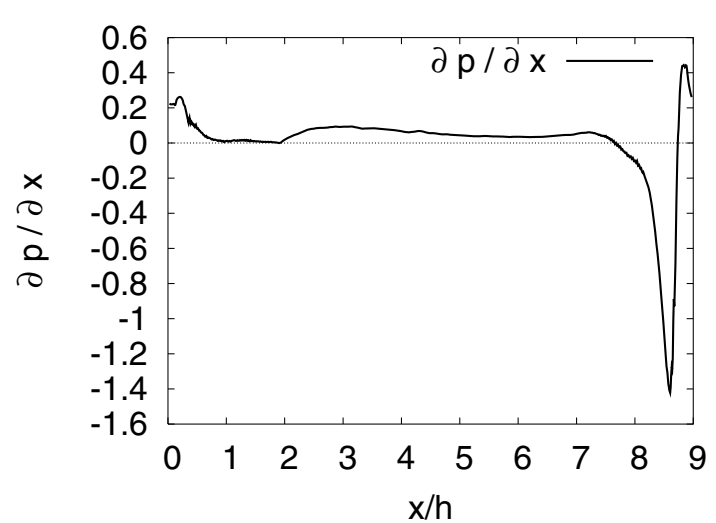

Figure 4. $R e=5600$; Pressure gradient along the bottom wall.

In the following the performace of our proposed scaling will be investigated. Starting with the traditional scaling for $U^{+}$and $y^{+}$problems arise in the regions where the wall shear stress and resp. $u_{\tau}$ becomes zero. Figure 6 shows the velocity scales plotted along the bottom wall. In [30,31] the flow has been categorized according to the parameter $\alpha$ which is a measure for the ratio between wall shear stress and pressure gradient. Figure 5 shows both $\alpha$ and $\frac{1}{2}(1-\alpha)^{3 / 2}$ plotted along the bottom wall. Table 2 gives an overview of the locations where $\alpha=0$ or $\alpha=1$ which is equivalent to $u_{\tau}=0$ or $u_{p}=0$, respectively. 


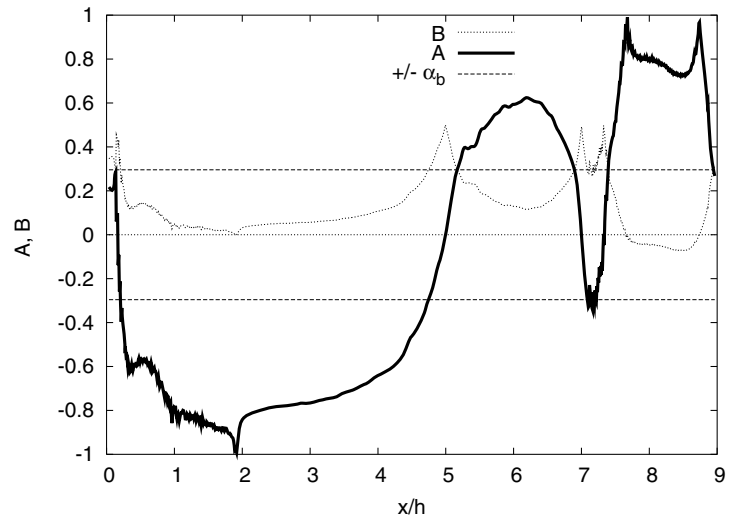

FiguRe 5. $R e=5600 ;$ Values for $\mathrm{A}$ and $\mathrm{B}$ (see equation (2.7)) plotted along the bottom wall.

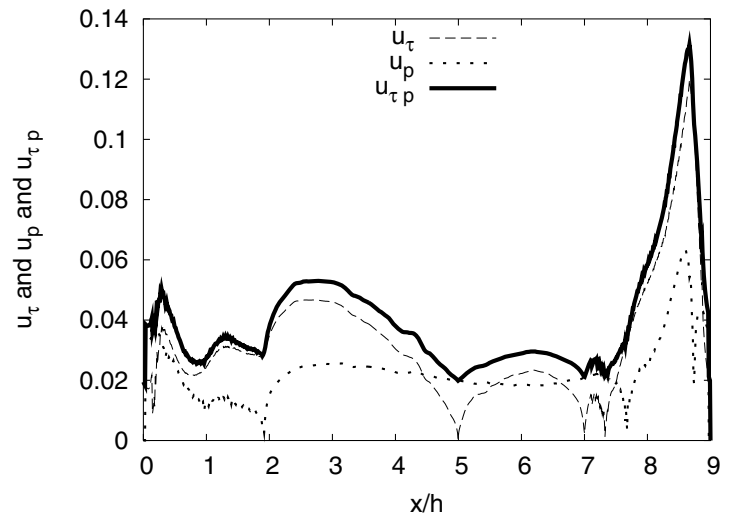

Figure 6. $R e=5600$; Comparison of velocity scales $u_{\tau}, u_{p}$ and $u_{\tau p}$

\begin{tabular}{|c|c|c|c|c|c|c|c|c|}
\hline & $x_{01}$ & $x_{02}$ & $x_{03}$ & $x_{04}$ & & $x_{11}$ & $x_{12}$ & $x_{13}$ \\
\hline \hline$\alpha=0:$ & 0.17 & 4.99 & 7.00 & 7.33 & $\alpha=1:$ & 1.91 & 7.66 & 8.74 \\
\hline
\end{tabular}

\begin{tabular}{|c|c|c|c|c||c|c|}
\hline & $x_{a 1}$ & $x_{a 2}$ & $x_{a 3}$ & $x_{a 4}$ & $x_{a 5}$ & $x_{a 6}$ \\
\hline \hline$x / h:$ & 0.25 & 1.9 & 3 & 5 & 6.98 & 7.35 \\
\hline$\alpha:$ & 0.4 & 0.97 & 0.75 & 0.12 & 0.19 & 0.19 \\
\hline $\operatorname{sign}\left(\tau_{w}\right):$ & - & - & - & + & + & + \\
\hline $\operatorname{sing}\left(\frac{\partial p}{\partial x}\right):$ & + & + & + & + & + & + \\
\hline
\end{tabular}

TABle 3. Positions in the flow with friction/pressure relation $\alpha$ (See Figure 2)

In what follows various positions are investigated that are characterized by typical $\alpha$ values. The positions (see Table 3) are indicated in Figure 2. We will first concentrate on the comparison between traditional scaling and proposed scaling (including pressure gradient) and second compare velocity profiles at different positions with similar $\alpha$ values. We consider positions within the recirculation zone and close to reattachment. The pressure gradient is positive for all four positions $\left(x_{a 1 \ldots a 4}\right)$. The first position $x_{a 1}(x / h=0.25)$ is shortly after separation $(x / h=0.17)$. This separation is induced by the curvature of the surface and the pressure gradient in streamwise direction. In Figure 4 the pressure gradient shows a local maximum close to $x_{a 1}$ which results in $\alpha=0.4$. In Figure 7, traditional wall scaling ( eq. 2.10) is compared with our proposed scaling ( eq. 2.7 ). This position is characterized by a very thin recirculation zone. The traditional near-wall prediction of the profile based on wall shear stress only would give negative velocities for all distances although the backflow is confined for $y^{+} \leq 1$. The proposed scaling ( eq. 2.7) is able to predict this thin recirculation by its quadratic term based on the local pressure gradient and consequently stays close to the DNS profile up to $y^{*}=5$. The result of the velocity profile scaled without pressure gradient can be understood when looking at Figure 2 . The thin layer of recirculation can be seen in Figure 7 where the model equation for the traditional scaling is not able to capture the change in flow direction from backflow to forward flow. On the other hand, scaling solely based on the pressure gradient would also be insufficient. The combination of both contributions as given in equation (2.7) is important. It can be seen from Figure 7 that the velocity profile described by equation (2.7) is much closer to the numerical result than the velocity profile without pressure gradient. 


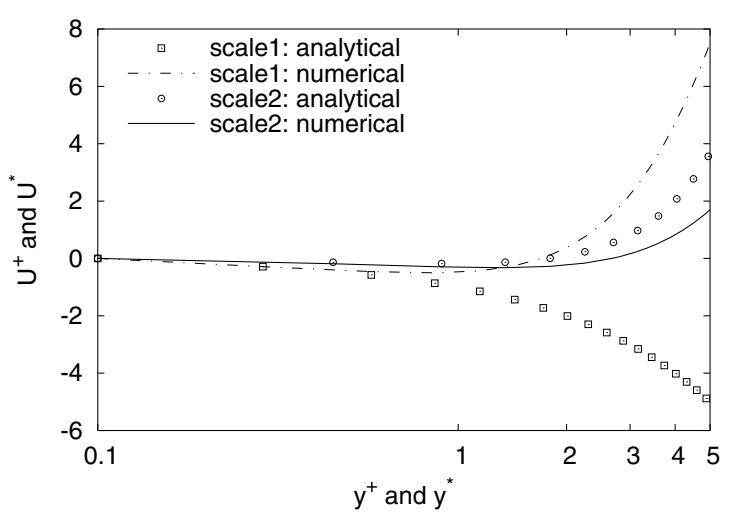

Figure 7. Averaged velocity profile at $x_{a 1}=0.25$; Scaling 1 (traditional, eq. (2.10)) and scaling 2 (proposed, eq. (2.7)); $(\operatorname{Re}=5600 ; \alpha \approx 0.4)$.

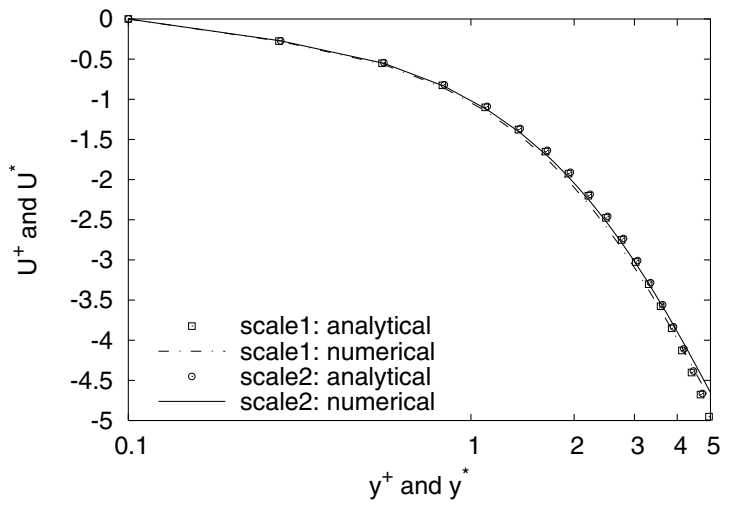

Figure 8. Averaged velocity profile at $x_{a 2}=1.9 ;$ Scaling 1(traditional, eq. (2.10)) and scaling 2(proposed, eq. (2.7)); $(\operatorname{Re}=5600 ; \alpha \approx 0.97)$.

Further down in the recirculation region $\left(x_{a 2}\right)$ the effect of the pressure gradient decreases. Figure 4 shows a local minimum of the pressure gradient which is close to zero. As a result the effect of the wall shear stress is dominant in equation (2.7) and the $\alpha$ coefficient is nearly one. Here our proposed scaling is equivalent to the traditional scaling. The pressure effect is negligible and the scaling is only based on the wall shear stress. Consequently, in Figure 8 both scalings fall on one curve. Downstream of $x_{a 2}$, still within the recirculation bubble, the effect of the pressure gradient increases again and at $x_{a 3} \alpha$ yields 0.75 . The proposed scaling still exhibits improved prediction as can be observed in Figure 9.

Close to reattachment at $x_{a 4}$ ( Figure 10 ) the pressure gradient becomes dominant $(\alpha \approx 0.12)$ which is responsible for the fast deviation of the traditional prediction from the DNS profile. Here again, pressure gradient based scaling stays closer at the DNS for larger wall distances. The fact that large deviations occur for $y^{*} \geq 3$ renders the importance of Reynolds stresses to compensate for the fast quadratic behaviour of the pressure gradient based term of equation (2.7). Note Figure 6 where the velocity and pressure scales are plotted in comparison to our combined pressure-velocity scale. An important observation is the fact that the combined pressure-velocity scale never reaches zero in the averaged flow field.

The following investigation discusses the observation that similar $\alpha$ values are observed in different flow situations which should lead to similar near-wall profiles. One example is given for two positions given in Table 3. Position $x_{a 5}$ and $x_{a 6}$ have similar $\alpha$ values. $x_{a 5}$ is located before the tiny recirculation region at $x / h \approx 7$ whereas $x_{a 6}$ is located after the separation bubble. In both cases the wall shear stress and the pressure gradient have the same sign and both cases can be directly compared. We should observe a similar near-wall profile in both cases. Figure 11 and Figure 12 confirms this assumption. This supports the assumption that we could observe universal velocity profiles for specific $\alpha$ values. 


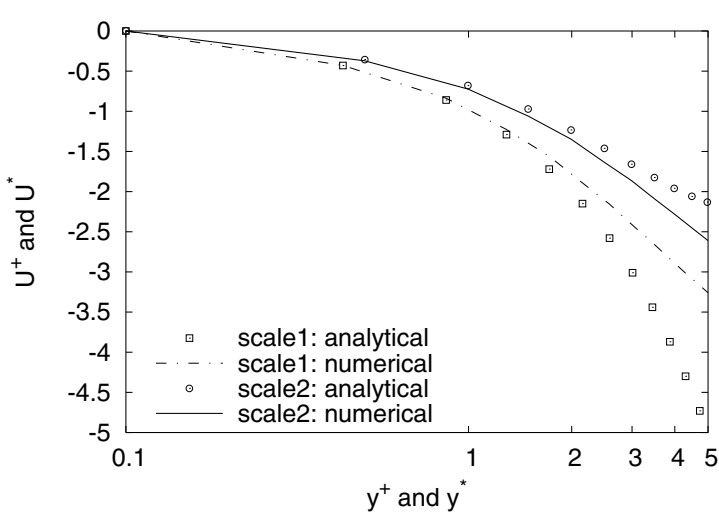

FIgure 9. Averaged velocity profile at $x_{a 3}=3 ;$ Scaling 1(traditional, eq. (2.10)) and scaling 2(proposed, eq. (2.7)); $(\operatorname{Re}=5600 ; \alpha \approx 0.75)$.

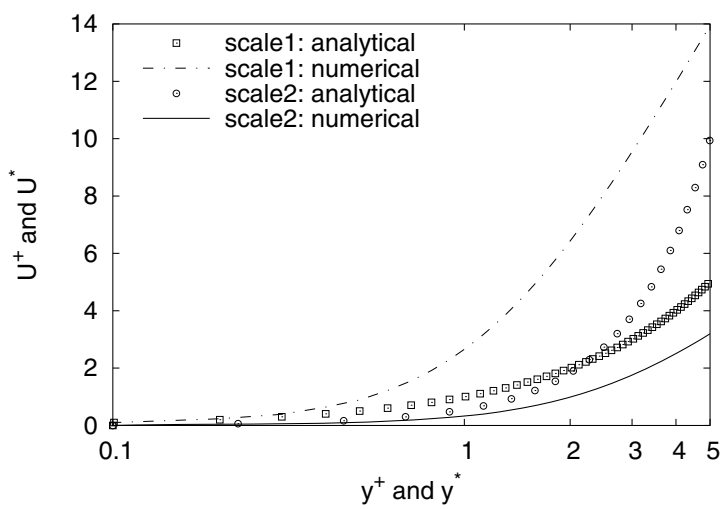

Figure 11. Averaged velocity profile at $x_{a 5}=6.98$; Scaling 1(traditional, eq. (2.10)) and scaling 2(proposed, eq. (2.7)); $(\operatorname{Re}=5600 ; \alpha \approx 0.19)$.

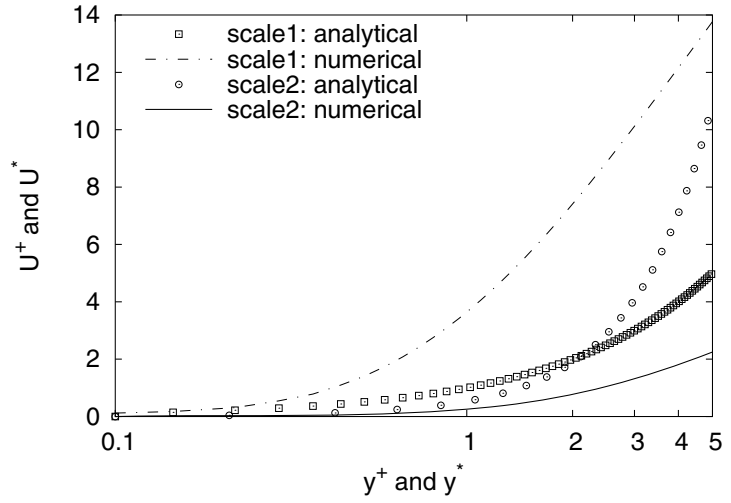

Figure 10. Averaged velocity profile at $x_{a 4}=5$; Scaling 1(traditional, eq. (2.10)) and scaling 2(proposed, eq. (2.7)); $(\operatorname{Re}=5600 ; \alpha \approx 0.12)$.

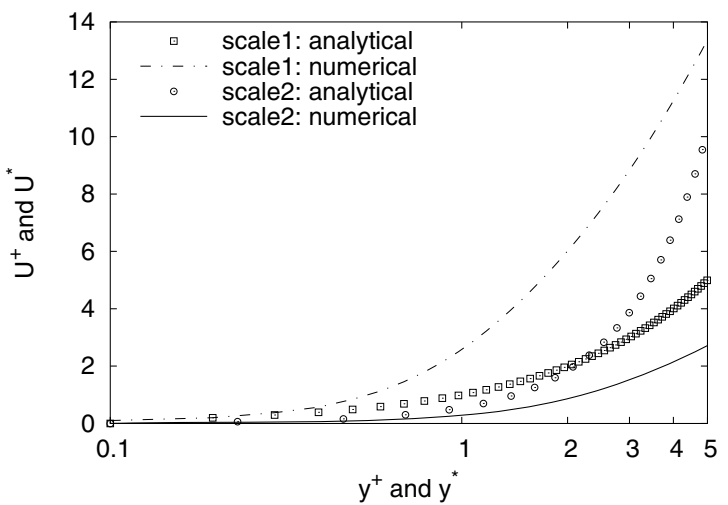

Figure 12. Averaged velocity profile at $x_{a 6}=7.35$; Scaling 1(traditional, eq. (2.10)) and scaling 2(proposed, eq. (2.7)); $(\operatorname{Re}=5600 ; \alpha \approx 0.19)$.

\subsection{Compressible channel flow}

The aim of the present section is to investigate the compressible flow separation in a plane channel with isothermal walls. The separation is artificially generated by applying a distributed force $g$ within the domain sketched in Figure 13 .

Both the inlet and the outlet boundary conditions must be prescribed for the domain 2. A fully developed periodical channel flow (domain 1) is used in order to ensure realistic inflow conditions, semi-reflecting outflow conditions are applied at the outlet.

We assume the fluid to be an ideal gas with ratio of specific heats $\gamma=1.4$, constant Prandtl number $\operatorname{Pr}=0.7$ and power-law temperature-dependent viscosity $\mu(T)=\mu\left(T_{w}\right)\left(\frac{T}{T_{w}}\right)^{0.7}$. We consider Mach numbers, based on 


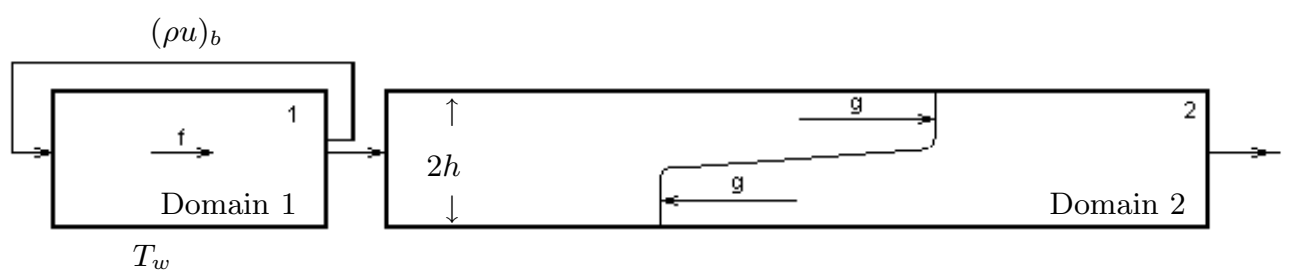

FiguRE 13. Geometry of the spatially developing channel flow

the bulk velocity and sound speed at the walls, of $M=\frac{(\rho u)_{b} / \rho_{b}}{\sqrt{\gamma R T_{w}}}=0.3$ to 5.0 and the Reynolds number, defined in terms of bulk mass flow, channel half-width and wall viscosity, of $R e=\frac{(\rho u)_{b} h}{\mu_{w}}=1200$.

The conservative non-dimensional compressible Navier-Stokes equations are numerically solved using fully explicit Mc Cormack scheme [32], modified by Gottlieb \& Turkel [33] to get second order accuracy in time and fourth order accuracy in space $[34,35]$.

\subsubsection{Periodical $2 D$ compressible channel flow}

We are seeking for a homogeneous velocity and density solution in the streamwise direction. For this purpose we have split the pressure and the temperature in a non-homogeneous contribution, due to the pressure drop into the channel and a homogeneous part, $P=P_{0}+p$ and $\theta=T_{0}+T$ respectively, in both the momentum and the total energy equations. Note that such demonstration was performed elsewhere for the incompressible flow in a channel with convection/rotation [36]. In the present case, the compressible non-dimensional Navier-Stokes equations are:

$$
\begin{array}{r}
\frac{\partial \rho}{\partial t}+\frac{\partial\left(\rho u_{i}\right)}{\partial x_{i}}=0 \\
\frac{\partial\left(\rho u_{i}\right)}{\partial t}+\frac{\partial}{\partial x_{j}}\left(\rho u_{i} u_{j}+\frac{1}{\gamma M^{2}} p \delta_{i j}-\frac{\mu}{R e} S_{i j}\right)=f \delta_{i 1} \\
\frac{\partial(\rho e)}{\partial t}+\frac{\partial}{\partial x_{j}}\left[\left(\rho e+p \delta_{i j}\right) u_{i}-\gamma M^{2} \frac{\mu}{R e} u_{i} S_{i j}-\frac{\gamma}{\gamma-1} \frac{\mu}{R e P r} \frac{\partial T}{\partial x_{j}}\right]=\gamma M^{2} \mathcal{W}
\end{array}
$$

with the total energy $\rho e=\frac{p}{\gamma-1}+\frac{\gamma M^{2}}{2} \rho u_{i}^{2}$ and the pressure $p=\rho T$. All quantities are non-dimensionalized by the wall temperature $T_{w}$, the channel half-width $h$, the bulk-averaged density $\rho_{b}$ and the bulk mass flux $(\rho u)_{b}$.

The source terms in the momentum and total energy equations are $f=\left.\frac{\mu_{w}}{R e} \frac{\partial u}{\partial y}\right|_{w}=-\frac{1}{\gamma M^{2}} \frac{\partial P_{0}}{\partial x}$ and $\mathcal{W}=$ $-\frac{1}{\gamma M^{2}} \frac{\gamma}{\gamma-1} u \frac{\partial P_{0}}{\partial x}=\frac{\gamma}{\gamma-1} u f$ respectively. Our strategy differs from the usual one in the literature which consists of viewing $f$ as an external force producing the work $\mathcal{W}=u f$ in the total energy equation [35]. In the present case the decrease of internal energy in the streamwise direction is not neglected [23].

The analytical solutions for the streamwise velocity and the temperature in a compressible channel flow write respectively

$$
\begin{aligned}
\frac{u(y)}{u_{b}} & =-\frac{3}{2} \frac{y^{2}-h^{2}}{h^{2}} \\
\frac{T(y)-T_{w}}{T_{w}} & =\frac{3}{8} \operatorname{Pr}^{2} \frac{y^{2}-h^{2}}{h^{2}} \frac{(3-2 \gamma) y^{2}-(3+2 \gamma) h^{2}}{h^{2}} \frac{\left(\rho_{b} u_{b}\right)^{2}}{(\rho u)_{b}^{2}}
\end{aligned}
$$




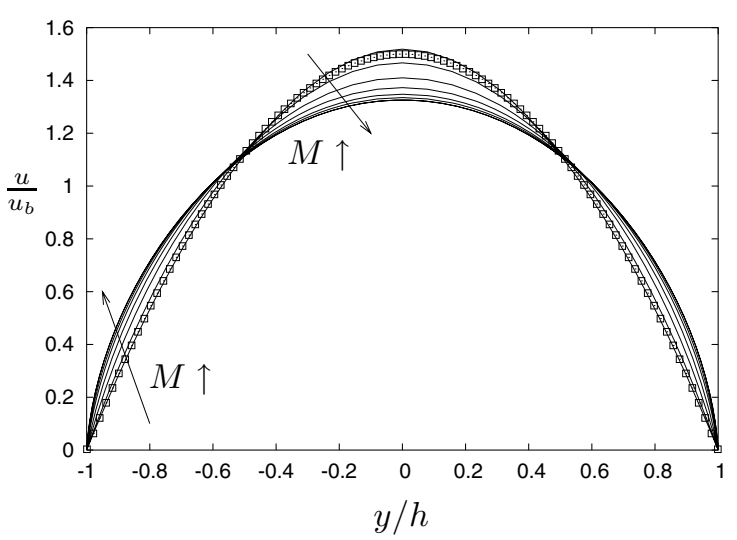

Figure 14. Profiles of streamwise velocity, $0.3 \leq M \leq 5.0$, $R e=1200$.

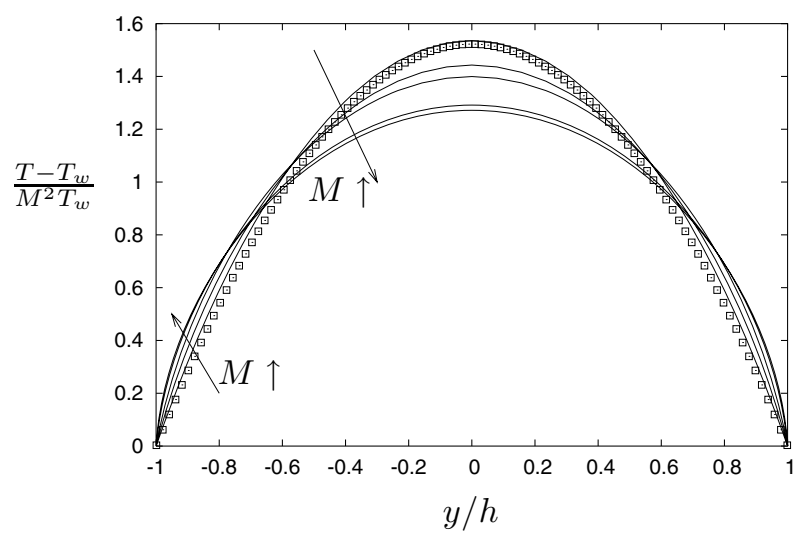

Figure 15. Profiles of temperature, $0.3 \leq M \leq 5.0, R e=1200$.

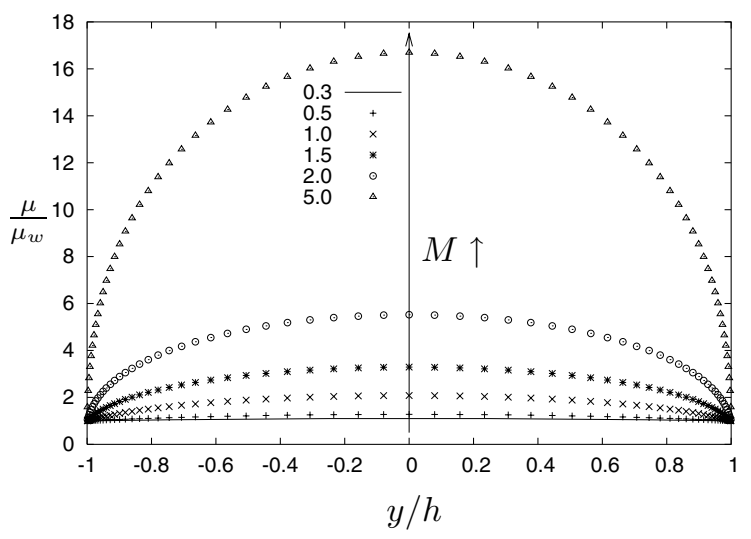

Figure 16. Profiles of dynamic viscosity, $0.3 \leq M \leq 5.0, R e=$ 1200

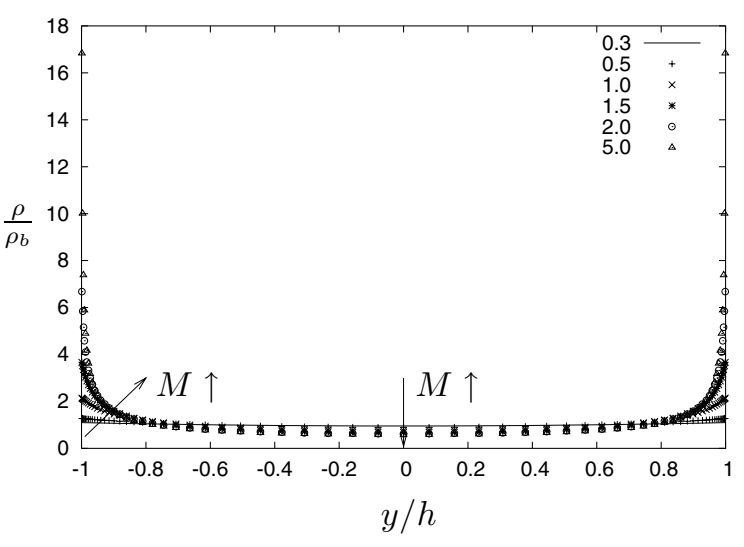

Figure 17. Profiles of density, $0.3 \leq M \leq 5.0, R e=1200$

where $u_{b}$ is the bulk velocity. Eqs. 3.40 and 3.41 are well satisfied even up to $M=5.0$ (figures 14 and 15 ). Figures 15, 16 and 17 show that the maximum temperature - and therefore the maximum dynamic viscosity and minimum density occur at the channel centerline. Furthermore, the maximum gradients of density, velocity and temperature are located near the walls and become increasingly steep as the Mach number increases. These sharp near-wall gradients are a very important attribute of non-adiabatic compressible boundary layers [24]. The profiles of local Mach number $M_{l}=\frac{u(y)}{\sqrt{\gamma R T(y)}}$ are given in figure 18. The variation with Mach number of the fluid properties causes the local Re number $R e_{l}=\frac{\rho(y) u(y) h}{\mu(y)}$ to change shape as the Mach number increases (figure 19). 


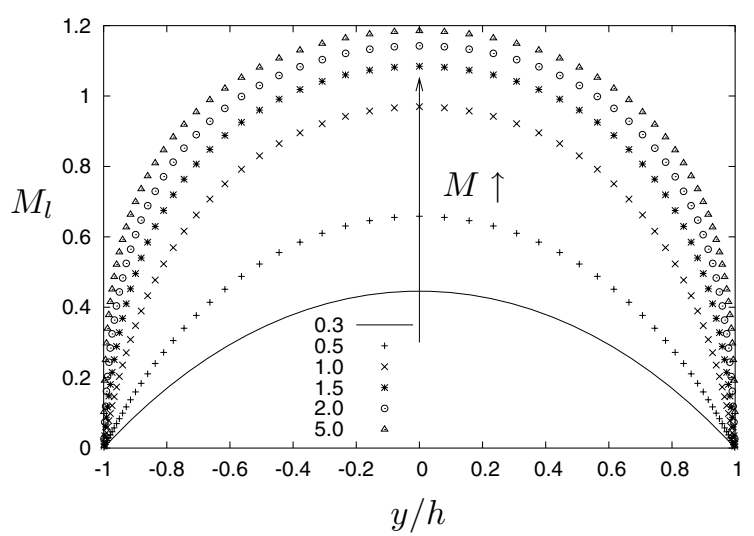

Figure 18. Profiles of local Mach number, $0.3 \leq M \leq 5.0$, $R e=1200$

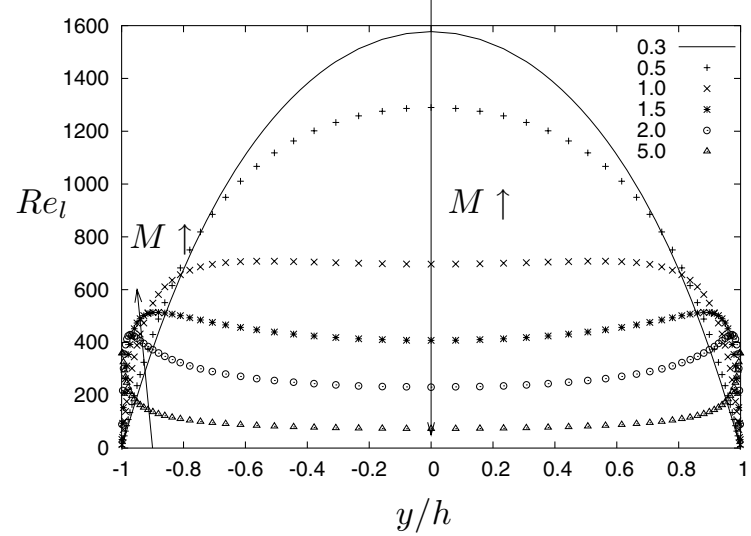

Figure 19. Profiles of local Reynolds number, $0.3 \leq M \leq$ 5.0, $R e=1200$

\subsubsection{Inflow and outflow boundary conditions}

For specifying boundary conditions, the characteristic analysis described in [37,38] for Euler equations and in [39] for both Euler and Navier-Stokes equations is used. The Navier-Stokes equations (3.37)-(3.39) are converted to an equivalent set of wave equations, which represent nonlinear waves propagating at characteristic velocities. They write in $2 \mathrm{D}$,

$$
\begin{aligned}
\frac{\partial \rho}{\partial t} & +d_{1}+\frac{\partial}{\partial y}(\rho v)=0 \\
\frac{\partial(\rho u)}{\partial t} & +u d_{1}+\rho d_{3}+\frac{\partial}{\partial y}(\rho u v)=\frac{1}{R e} \frac{\partial\left(\mu S_{1 j}\right)}{\partial x_{j}} \\
\frac{\partial(\rho v)}{\partial t} & +v d_{1}+\rho d_{4}+\frac{\partial}{\partial y}\left(\rho v v+\frac{1}{\gamma M^{2}} p\right)=\frac{1}{R e} \frac{\partial\left(\mu S_{2 j}\right)}{\partial x_{j}} \\
\frac{\partial(\rho e)}{\partial t} & +\gamma M^{2}\left[\frac{1}{2}\left(u_{k} u_{k}\right) d_{1}+\frac{d_{2}}{\gamma-1}+\rho u d_{3}+\rho v d_{4}\right]+\frac{\partial}{\partial y}[(\rho e+p) v] \\
& =\frac{\partial}{\partial x_{j}}\left(\gamma M^{2} \frac{\mu}{R e} u_{i} S_{i j}+\frac{\gamma}{\gamma-1} \frac{\mu}{R e P r} \frac{\partial T}{\partial x_{j}}\right)
\end{aligned}
$$

Since only the 2D case is presently discussed, the characteristic velocity $\left(\lambda_{4}\right)$, the wave amplitude $\left(L_{4}\right)$ and the derivative $\left(d_{4}\right)$ specific to the $3 \mathrm{D}$ case [39] are not considered here. The characteristic velocities are given as the solution to the eigenvalue problem and are $\lambda_{1}=u-c_{n o r m}, \lambda_{2}=\lambda_{3}=u, \lambda_{5}=u+c_{n o r m}$, where $c_{n o r m}=\frac{1}{\sqrt{\gamma} M} c$ is the normalized speed of sound, and $p_{\text {norm }}=\frac{1}{\gamma M^{2}} p$ is the normalized pressure.

The system of Eqs. (3.42)-(3.45) includes derivatives normal to the $x=0$ or $L_{x}$-boundary $\left(d_{1}\right.$ to $\left.d_{4}\right)$, derivatives 
parallel to the $x=0$ or $L_{x}$-boundary like $\left(\frac{\partial}{\partial y}\right)(\rho v v)$ and local viscous terms. The vector $\mathbf{d}$ is expressed as

$$
\mathbf{d}=\left(\begin{array}{c}
\frac{1}{c_{\text {norm }}^{2}}\left[L_{2}+\frac{1}{2}\left(L_{5}+L_{1}\right)\right] \\
\frac{1}{2}\left(L_{5}+L_{1}\right) \\
\frac{1}{2 \rho c_{\text {norm }}}\left(L_{5}-L_{1}\right) \\
L_{3}
\end{array}\right)=\left(\begin{array}{c}
\frac{\partial(\rho u)}{\partial x} \\
\frac{\partial}{\partial x}\left(c_{\text {norm }}^{2} \rho u\right)+(1-\gamma) u \frac{\partial p_{\text {norm }}}{\partial x} \\
u \frac{\partial u}{\partial x}+\frac{1}{\rho} \frac{\partial p_{\text {norm }}}{\partial x} \\
u \frac{\partial v}{\partial x}
\end{array}\right)
$$

where the $L_{i}$ 's are the amplitudes of characteristic waves

$$
\begin{aligned}
L_{1} & =\lambda_{1}\left(\frac{\partial p}{\partial x}-\rho c_{\text {norm }} \frac{\partial u}{\partial x}\right) & L_{2} & =\lambda_{2}\left(c_{\text {norm }}^{2} \frac{\partial \rho}{\partial x}-\frac{\partial p}{\partial x}\right) \\
L_{3} & =\lambda_{3} \frac{\partial v}{\partial x} & L_{5} & =\lambda_{5}\left(\frac{\partial p}{\partial x}+\rho c_{\text {norm }} \frac{\partial u}{\partial x}\right)
\end{aligned}
$$

The advancement in time of variables on the boundary is made using the system of Eqs. (3.42)-(3.45). For a subsonic inlet, the only characteristic $L_{1}$ propagating against the flow is computed using Eqs. (3.47) and the other characteristic waves, propagating in the flow direction, are specified using both the amplitude variations $L_{i}^{p}$ in the periodical channel and the pressure drop contribution $\left(-\frac{\partial p_{\text {norm }}}{\partial x}=f\right)$ :

$$
\begin{aligned}
L_{2}^{s} & =L_{2}^{p}+u f \\
L_{3}^{s} & =L_{3}^{p} \\
L_{5}^{s} & =L_{5}^{p}-\left(u+c_{\text {norm }}\right) f
\end{aligned}
$$

The only viscous condition is $\frac{\partial\left(\mu S_{11}\right)}{\partial x}=0$.

Considering a subsonic outlet, the characteristic waves leaving the domain, $L_{2}, L_{3}, L_{5}$, are computed from

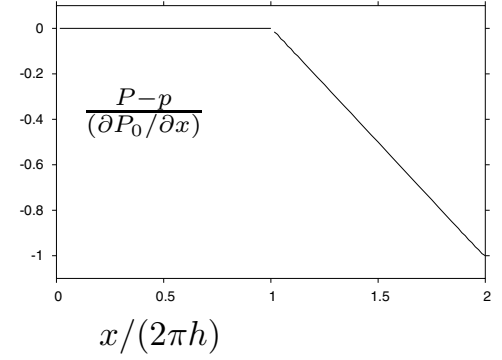

FIGURE

20. Pressure evolution with respect to streamwise direction

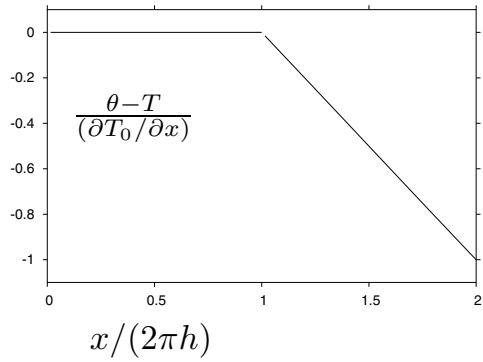

Figure

21. Temperature evolution with respect to streamwise direction

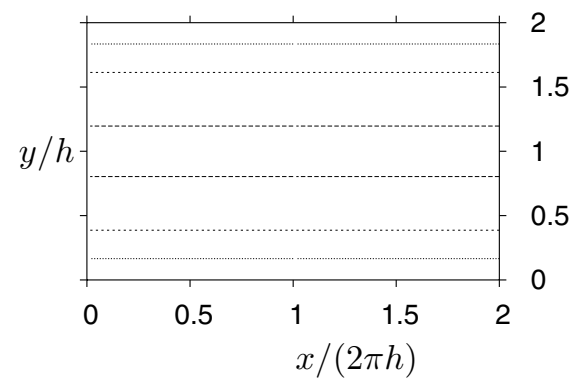

Figure 22. Velocity isovalues for the reference case

their definitions in (3.47) and the incoming wave $L_{1}$ is specified using the Rudy and Strikwerda's model [40] which was further adapted for channel flows

$$
L_{1}=-\frac{k\left(u^{2}-c_{\text {norm }}^{2}\right)}{c_{\text {norm }}} f
$$


where the constant $k$ is set to $\frac{1}{1+M}$. The viscous conditions are $\frac{\partial\left(\mu S_{12}\right)}{\partial x}=0, \frac{\partial\left(\mu S_{13}\right)}{\partial x}=0, \frac{\partial}{\partial x}\left(\mu \frac{\partial T}{\partial x}\right)=0$. The case of a spatially developing channel flow for $M=0.4$ and $R e=1200$ is presently considered. Figures (20) and (21) show the expected distribution of the pressure and the temperature in the spatially developing channel, $\frac{P-p}{\left(\partial P_{0} / \partial x\right)} \approx-\frac{x}{2 \pi h}$ and $\frac{\theta-T}{\left(\partial T_{0} / \partial x\right)} \approx-\frac{x}{2 \pi h}$ respectively. The correct connection at $x / 2 \pi h=1.0$ between periodical and spatial developing channel is obtained in terms of velocity (figure 22) and temperature.

\subsubsection{Separating compressible flow}

The separation of the flow in the channel is generated by applying a distributed force $g$ within the domain of an equilibrium channel flow and leaving the flow readjust to the new conditions. This distributed force per unit area is equivalent to a locally adverse pressure gradient $\left(\frac{\partial p}{\partial x}>0\right)$ and has the expression [41]

$$
g(x, y)=-90 f \sin \left(2 \pi \frac{x}{L_{x}}\right) \tanh \left(5 \frac{y}{h}\right)
$$

We present the results for a separating compressible channel flow at the Mach number of 0.4 and the Reynolds number of 1200. The separation and reattachment points, $\frac{x}{2 \pi h}=4.6$ and 7.0 respectively, are identified from figure 25 since the negative values of $\alpha$ ratio mark the recirculation bubble. In conformity with velocity isovalues shown in figure 23, behind the recirculation bubble the flow relaxes back towards steady channel flow.

Figure 24 shows that the temperature field in the lower half of the channel is less perturbated than the velocity

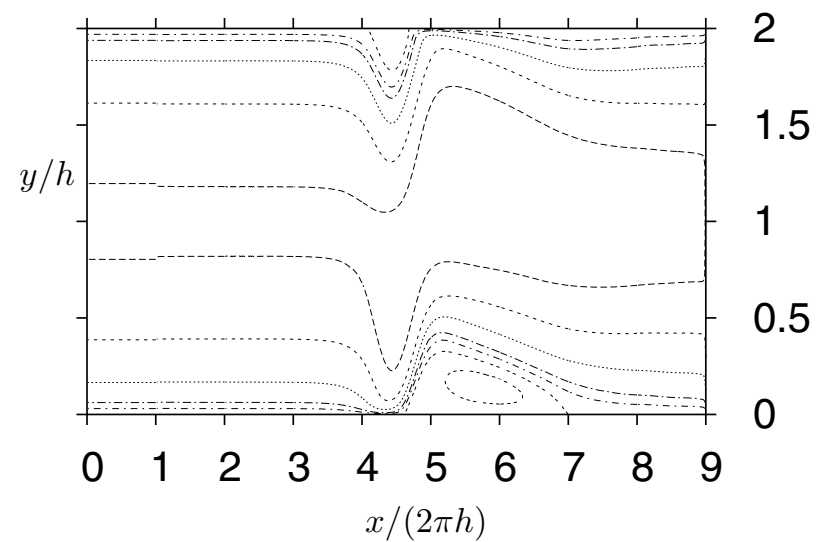

Figure 23. Velocity isovalues for the separating flow

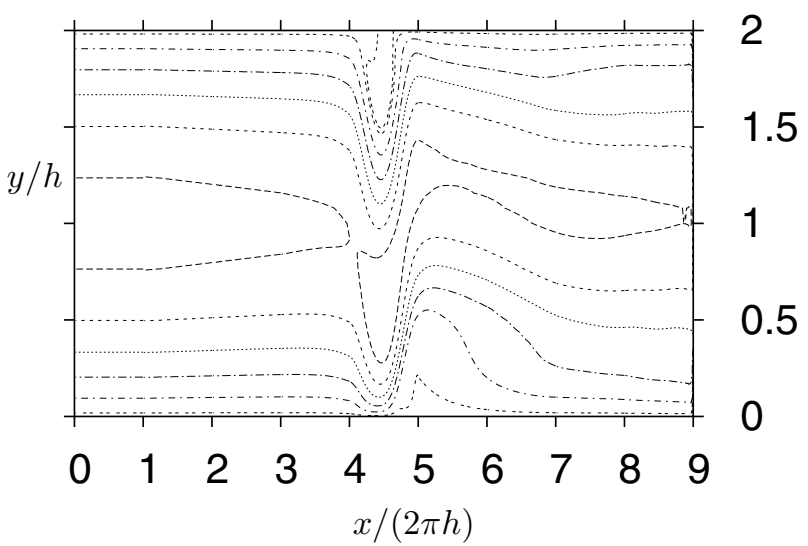

Figure 24. Temperature isovalues for the separating flow

field. This is confirmed by the values of the $\beta$ ratio which remain about $\beta=1$ in the full channel (Figure 26 ). Using the non-dimensional system of Eqs. (3.37)-(3.39) based on Reynolds and Mach numbers (and presently referred to with the adim superscript), the equations (2.5) and (2.25) write

$$
\alpha=\frac{\left(u_{\tau}^{\text {adim }}\right)^{2}}{\left(u_{\tau}^{a d i m}\right)^{2}+\frac{R e^{1 / 3}}{\left(\gamma M^{2}\right)^{2 / 3}}\left(u_{p}^{a d i m}\right)^{2}} \quad \beta=\frac{T_{\tau}^{a d i m}}{T_{\tau}^{a d i m}+\frac{(\gamma-1) M^{2 / 3}}{2(\gamma R e)^{2 / 3}}\left(u_{p}^{a d i m}\right)^{2}}
$$

For the relatively low Mach number $M=0.4$ and the Reynolds number $R e=1200$ presently considered, the effects of the pressure gradient $\frac{\partial p}{\partial x}$ are expected to be weak on the $\beta$ ratio while they are strong on the $\alpha$ ratio. The profiles of the averaged velocity and the averaged total temperature at $x /(2 \pi h)=4.98$ are given in 


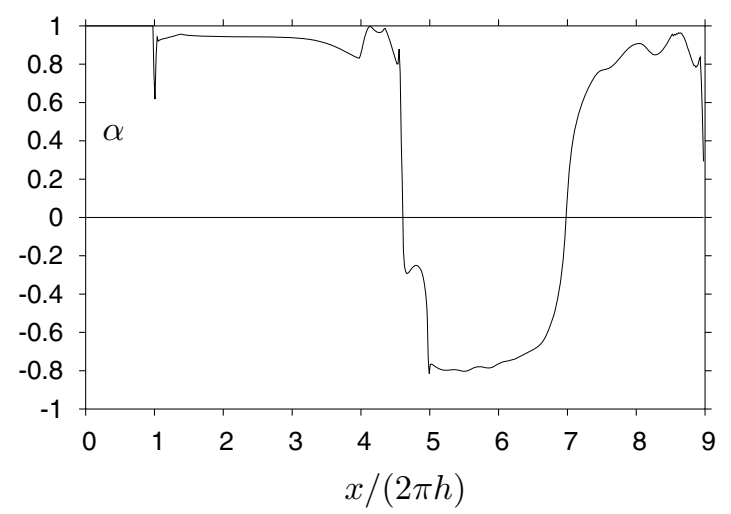

Figure 25. Values for $\alpha$ ratios plotted along the bottom walls

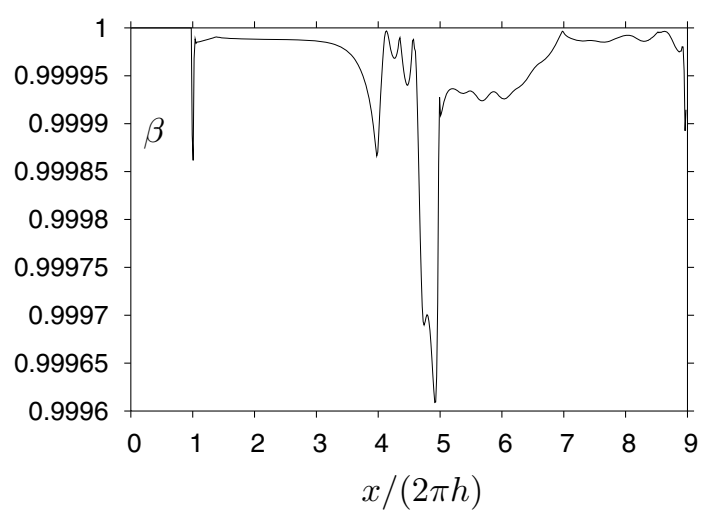

Figure 26. Values for $\beta$ ratios plotted along the bottom walls

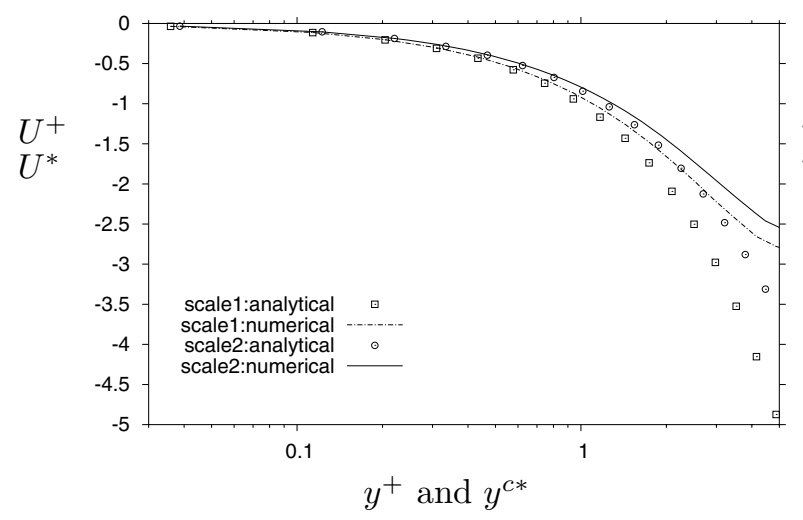

Figure 27. Averaged velocity profile at $\frac{x}{2 \pi h}=4.98$; Scaling 1 (traditional, eq. (2.19)) and scaling 2 (proposed, eq. $(2.28)) ;(R e=1200, M=0.4$; $\alpha \approx 0.8, \beta \approx 1$ )

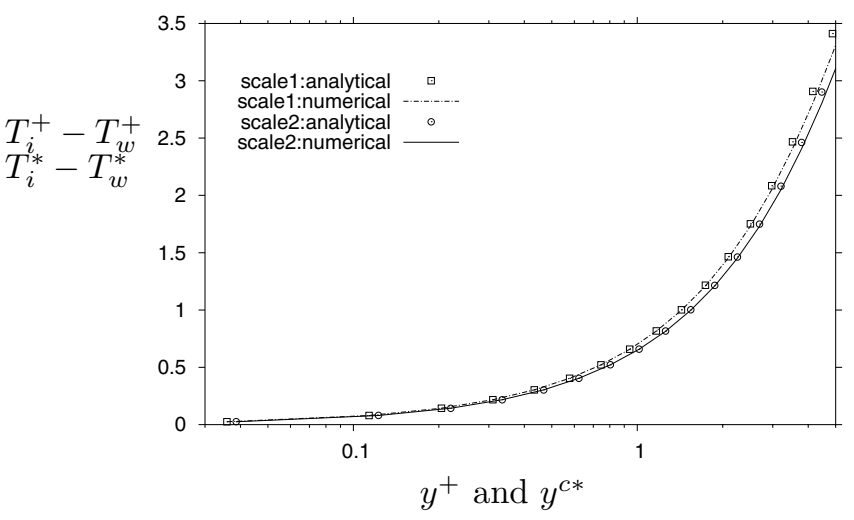

Figure 28. Averaged total temperature profile at $\frac{x}{2 \pi h}=4.98$; Scaling 1 (traditional, eq. (2.20)) and scaling 2 (proposed, eq. (2.32)); $(R e=1200$, $M=0.4 ; \alpha \approx 0.8, \beta \approx 1$ )

Figures 27 and 28 respectively. According to Eq. 2.32, the temperature in the lower half of the channel is a linear function of $y$. 


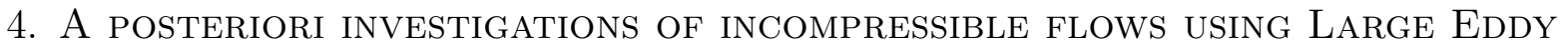 Simulation (LES)}

We performed LES of the channel flow with periodic hill constrictions as introduced in section 3 . The properties of the non-equidistant cartesian grid used for the simulations are given given in Table 4 . The viscous region is not completely resolved, but it remains in a range that is accepted as linear region in most explicit wall models. By using this grid resolution the simplified near-wall equation developed in section 2.1 can be tested. This is achieved by comparing simulations with and without wall model. The simulations performed and their parameters are given in Table 5. Simulation S1 is the reference DNS. Simulation S2 uses a wall model formulation for the linear region of the flow and Simulation S3 uses the same formulation including pressure gradient effects according to equation (2.7). The wall model implementation follows Tessicini et al. [42]. Computing time for the LES $\left(3.8 \cdot 10^{6}\right.$ grid cells) were 8 seconds per time step on a 2 processor Itanium PC. The DNS $\left(233 \cdot 10^{6}\right.$ grid cells) needed 7 seconds per time step on 32 computing nodes with 8 processors each on the Hitachi SR 8000 of the Leibniz computing center in Munich.

TABLE 4. LES grid: resolution given for position of maximum wall shear stress

\begin{tabular}{|l|l|l|l|l|l|l|l|}
\hline Re-number & $\Delta x^{+}$ & $\Delta y^{+}$ & $\Delta z^{+}$ & $n x$ & $n y$ & $n z$ & $n x * n y * n z$ \\
\hline 5600 & 12 & 29 & 11 & 246 & 102 & 154 & $3.8 \cdot 10^{6}$ \\
\hline
\end{tabular}

TABLE 5. Performed DNS/LES simulations at $R e=5600$

\begin{tabular}{|l|l|l|l|l|}
\hline & name & description & $\begin{array}{l}\text { separation } \\
\mathrm{x} / \mathrm{h}\end{array}$ & $\begin{array}{l}\text { reattachment } \\
\mathrm{x} / \mathrm{h}\end{array}$ \\
\hline S1: & REF-DNS & reference DNS & 0.16 & 4.87 \\
S2: & WM & wall model without dpdx & 0.36 & 4.25 \\
S3: & WM-DPDX & wall model including dpdx & 0.36 & 4.58 \\
\hline
\end{tabular}

Comparisons between simulation S2 (WM) and S3 (WM-DPDX) are shown in Figure 29 and Figure 30 for the streamwise velocity component at two different positions in the flow. Figure 29 compares the mean streamwise velocity at $x / h=0.05$ to the DNS data used for the a priori analysis. Simulation S2 with the simple wall model shows an overprediction of the velocity gradient. Including the pressure gradient formulation in our wall model simulation S3 improves the results. The average streamwise velocity profile follows the DNS data closely. A similar behaviour can be observed for the velocity profile at $x / h=8$. This is a position close to the wall shear stress maximum $(x / h \approx 8.7)$. The wall model implementation with pressure gradient used in Simulation S3 is an improvement to Simulation S2. Most striking is the improvement of the gradient of the streamwise velocity at $x / h=0.05$ close to the hill top. The wall model without pressure gradient is not able to capture the slope of the average profile. This confirms a priori investigations which have shown that velocity profiles at this position show much better agreement with the proposed scaling. In addition to that the improved slope of the velocity profile at $x / h=0.05$ could be a result from the improved near-wall prediction at $x / h=8$ ( Figure 30) since it influences the formation of the shear layer and as a result the flow properties at the hill top.

\section{Conclusions}

A modified law of the wall for the viscous sublayer has been introduced which accounts for the contributions of the pressure gradient in incompressible and compressible flows. Two parameters $\alpha$ and $\beta$ define the ratio of wall friction to pressure gradient and wall heat flux to pressure gradient, respectively. Dependent on these parameters the analytical velocity and temperature profiles are derived from simplified boundary layer equations. The equation for the velocity profile is similar in compressible and incompressible case. It yields a linear 


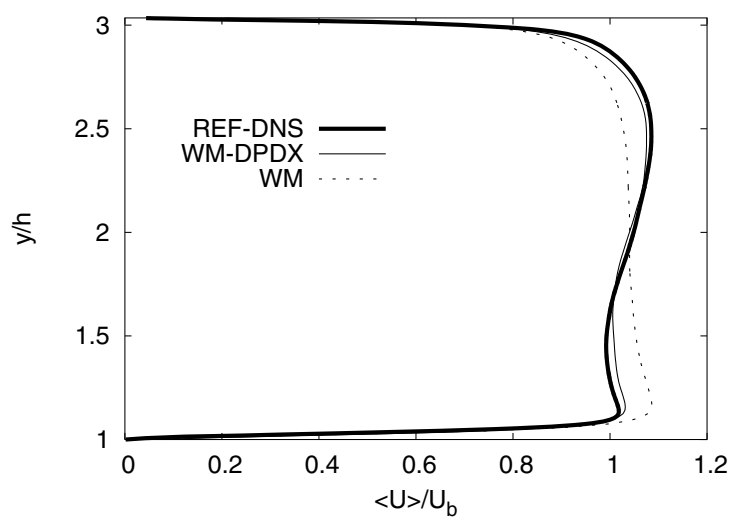

Figure 29. $R e=5600 ;$ Average streamwise velocity profile at $x / h=0.05$.

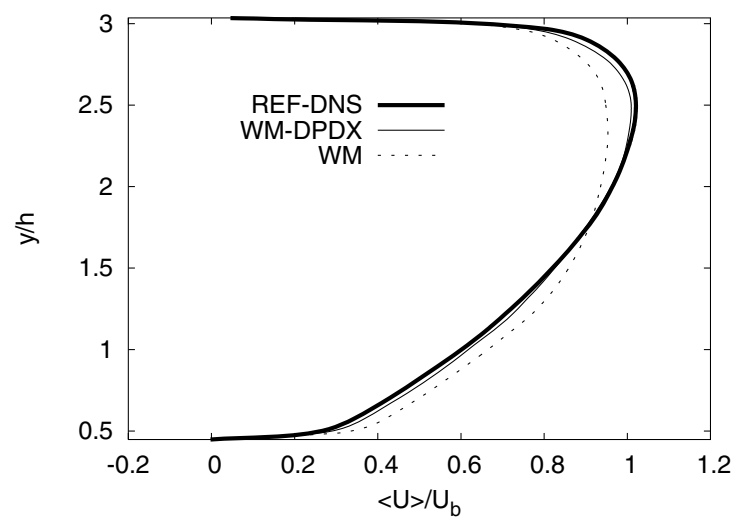

Figure 30. $R e=5600 ;$ Average streamwise velocity profile at $x / h=8$.

contribution based on the effect of wall shear stress and a parabolic contribution based on the pressure gradient effects. The only difference is that in the compressible case an integral length scale $y^{c *}$ is defined which accounts for viscosity and density changes in the boundary layer. The temperature law of the wall as a function of $y^{c *}$ is linear, parabolic and to the power of four dependent on the contributions of the friction, wall heat flux and pressure gradient. A priori analysis of the scaling yields good results in both types of flows, although the present $2 \mathrm{D}$ compressible laminar case is only considered to perform a preliminary study. Note that the parameters $\alpha=0$ and $\beta=0$ are especially useful to characterize positions in the flow where the wall friction and the wall heat flux are zero. A posteriori investigations show improvement in LES of incompressible channel flow with constrictions when using wall model formulations including pressure gradient effects.

\section{Acknowledgments.}

The present work was performed within the framework of the French-German research initiative 'Large Eddy Simulation of Complex Flows'. The authors greatfully acknowledge the financial support from the Deutsche Forschungsgemeinschaft (DFG), the Centre National de la Recherche Scientifique (CNRS) and the Centre d'été Mathématique de Recherche Avancée en Calcul Scientifique (CEMRACS)

\section{BIBLIOGRAPHY}

\section{REFERENCES}

[1] P. Sagaut. Large eddy simulation for incompressible flows - an introduction. Springer-Verlag, Berlin, second edition edition, 2002.

[2] F.H. Bark S. Zahrai and R.I. Karlsson. On anisotropic subgrid modeling. Eur. J. Mech. B/Fluids, 14(4):459-486, 1995.

[3] S. Zahrai, F.H. Bark, and R.I. Karlsson. On anisotropic subgrid modeling. Eur. J. Mech. B/Fluids, 14(4):459-486, 1995.

[4] U. Piomelli. High Reynolds number calculation using the dynamic subgrid-scale stress model. Phys. Fluids A, 5(6):1484-1490, June 1993.

[5] D.R. Chapman. Computational aerodynamics development and and outlook. AIAA Journal, 17(12):1293-1313, 1979.

[6] Hans-Jakob Kaltenbach. A priori testing of wall models for separated flows. Phys. Fluids., 15(10):3048-3068, 2003.

[7] R. Ruderich and H.H. Fernholz. An experimental investigation of a turbulent shear flow with separation, reverse flow, and reattachment. J. Fluid Mech., 163:283-322, 1986.

[8] E.W. Adams, J.P. Jahnston, and J.k. Eaton. Experiments on the structure of turbulent reattaching flow. Tech. Rep. MD-43, Thermosciences Division, Department of Mechanical Engineering, Stanford University, Stanford, CS, 1984.

[9] W.J. Devenport and E.P. Sutton. Near-wall behaviour of separated ad reattaching flows. AIAA J., 29(25), 1991.

[10] R.L. Simpson, Y.T. Chew, and B.G. Shivaprasad. The structure of a separating turbulent boundary layer. Part 1. mean flow and Reynolds stresses. J. Fluid Mech., 113:23-51, 1981. 
[11] B.S. Stratford. The prediction of separation of the turbulent boundary layer. J. Fluid Mech., 5:1-16, 1959.

[12] B.S. Stratford. An experimental flow with zero skin friction throughout its region of pressure rise. J. Fluid Mech., 5:17-35, 1959.

[13] F.M. White. Viscous fluid flow. Mc Graw-Hill, 1974.

[14] M. Skote, D.S. Henningson, and R.A.W.M. Henkes. Direct numerical simulation of self-similar turbulent boundary layers in adverse pressure gradients. Flow, Turbulence and Combustion, 60:47-85, 1998.

[15] M. Manhart and R. Friedrich. DNS of a turbulent boundary layer with separation. International Journal of Heat and Fluid Flow, 23(5):572-581, 2002.

[16] R.-L. Simpson. A model for the backflow mean velocity profile. AIAA J., 21:142, 1983.

[17] P. Bradshaw. Compressible turbulent shear layers. Annu. Rev. Fluid. Mech., 9:33-54, 1977.

[18] Michel, Quemard, and Durand. ONERA. N.T., 1969.

[19] J.F. Debieve, P. Dupont, D.R. Smith, and A.J. Smits. Supersonic turbulent boundary layer subjected to step changes in wall temperature. AIAA J., 35(1):51-57, 1997.

[20] Huang P.G. and Coleman G.N. Vand driest transformation and compressible wall-bounded flows. AIAA Paper, $305,1995$.

[21] C. Brun, M. Haberkorn, and P. Comte. Compressibility effects in fully developed channel flow up to mach $m=5$. Toulouse, 2003. Euromech Fluid Mechanics Conference.

[22] M. Haberkorn. LES d'écoulement compressible en canal plan : effets de compressibilité et propagation acoustique. PhD thesis, Université de Strasbourg, PhD, 2004.

[23] C. Brun, M. Petrovan, M. Haberkorn, and P. Comte. Universality of the structure of the turbulence in compressible isothermalwall channel flow. Theoretical and Computational Fluid Dynamics, 2006.

[24] G.N. Coleman, J. Kim, and R.D. Moser. A numerical study of turbulent supersonic isothermal-wall channel flow. J. Fluid Mech., 305, 1995.

[25] C. P. Mellen, J. Frhlich, and W. Rodi. Large-eddy simulation of the flow over periodic hills. In 16th IMACS World Congress, Lausanne, Switzerland, 2000.

[26] N. Peller and M. Manhart. Turbulent channel flow with periodic hill constrictions. In Arbeitsgemeinschaft Strömung mit Ablösung (STAB), Mitteilungen, pages 178-179, 2005.

[27] J. Fröehlich, C. P. Mellen, W. Rodi, L. Temmerman, and M. Leschziner. Highly resolved large-eddy simulation of separated flow in a channel with streamwise periodic constrictions. J. Fluid Mech., 526:19-66, 2005.

[28] N. Peller and M. Manhart. Dns of channel flow with periodic hill constrictions. In Gesellschaft für angewandte Mathematik und Mechanik (GAMM), 2004.

[29] M. Manhart, F. Tremblay, and R. Friedrich. MGLET: a parallel code for efficient DNS and LES of complex geometries. In Jensen et al., editor, Parallel Computational Fluid Dynamics 2000, pages 449-456, Amsterdam, 2001. Elsevier Science B.V.

[30] N. Peller, C. Brun, and M. Manhart. Wall layer investigations of channel flow with periodic hill constrictions. In DLES 6, Poitiers, September 12.-14. 2005.

[31] N. Peller, M. Manhart, T. Goossens, M. Petrovan, and C. Brun. Near-wall behaviour of a family of separating turbulent shear flows. In Euromech, Dresden, October 2005.

[32] R. Mc Cormack. The effect of viscosity in hypervelocity cratering. AIAA Paper, 69:354, 1969.

[33] D. Gottlieb and E. Turkell. Dissipative two-four methods for time dependent problems. Mathematics of Computation, 703:72330, 1976.

[34] F. Ducros, P. Comte, and Lesieur M. Large-eddy simulation of transition to turbulence in a boundary layer developing spatially over a flat plate. J. Fluid Mech., 526:19-66, 1996.

[35] M. Salinas Vázquez and O. Métais. Large-eddy simulation of the turbulent flow through a heated square duct. J. Fluid Mech., 201:238-453, 2002.

[36] J. Pallares and L. Davidson. Large-eddy simulations of turbulent heat transfer in stationary and rotating square ducts. Phys. Fl., 2804(2816):14(8), 2002.

[37] K.W. Thompson. Time dependent boundary conditions for hyperbolic systems. J. Comp. Phys., 1:24-68, 1986.

[38] K.W. Thompson. Time-dependent boundary conditions for hyperbolic systems ii. J. Comp. Phys., 89:439-461, 1990.

[39] T.J. Poinsot and S.K. Lele. Boundary conditions for direct simulations of compressible viscous flows. J. Comp. Phys., 103:16-42, 1992.

[40] D.H. Rudy and J.C. Strikwerda. A nonreflecting outflow boundary condition for subsonic navier-stokes calculations. J. Comp. Phys., 55(70):36, 1980.

[41] R.J.A. Howard. Direct numerical simulation and modelling of turbulent channel flows subjected to complex distortions. PhD thesis, University of London, Queen Mary and Westfield College, Mile End Road, London, E1 4NS, U.K., January 1999.

[42] F. Tessicini, G. Iaccarino, M. Fatica, M. Wang, and R. Verzicco. Wall modeling for large-eddy simulation using an immersed boundary method. Center of Turbulence Research, Annual Research Briefs, pages 181-187, 2002. 